\title{
Resolving conflicts between locality and anti-locality: Evidence from Luganda and Haya
}

\author{
Kenyon Branan*
}

\begin{abstract}
It has been proposed that grammars generally prefer to make the shortest possible move [Shortest], given the choice between two or more movers. It has also been proposed that there are general bans on movement which is in some sense too short [anti-locality]. What happens when the shortest move is too short? In this paper, I argue that elements which cannot move as a result of anti-locality are rendered irrelevant for Shortest, and show that this provides a novel account of patterns of symmetry and asymmetry in Luganda and Haya passives. There we will see a curious pattern: internal arguments may move across no more than one other postverbal argument. The theory developed leads to a simple explanation of these effects. Movement of one element across another indicates that the crossed element is too close to the landing site to undergo movement; but given a particular definition of anti-locality, only one element may be anti-local to a given landing site.
\end{abstract}

Keywords. syntax; locality; passive; anti-locality; Bantu

1. Introduction. It is often assumed that movement is subject to locality restrictions, which require movement not to be 'too long', defined in a variety of ways. Apparent violations of locality in movement are commonly attributed to successive cyclic movement, where multiple locality-respecting operations give the surface appearance of a locality violation. Theories of long distance $w h$-movement like those proposed in Chomsky (1973) and many others since, for instance, propose that wh-movement is clause bounded-wh-movement which crosses a clause boundary on these views is too long, and ruled out by a principle of locality. Cases of apparent cross-clausal $w h$-movement poses a problem for these theories; the solution to this problem is to say that apparent cases of cross-clausal movement involve multiple instances of movement which fit the definition of 'clause bound'.

A similar approach has been taken for cases of apparent violations of Shortest in A-movement [McGinnis (1998); Doggett (2004), a.o.], which will be spelled out in more detail in the first part of $\S 2$. Shortest is a principle that allows the grammar to decide which element to move to a particular position when there is more than one eligible target; here movement of the 'closest' target is preferred. The move made by these authors is the same: multiple instances of Shortest respecting movement can give rise to what appears on the surface to be a violation of Shortest, in much the same way multiple instances of clause-bound movement can give the surface appearance of a cross-clausal dependency.

This paper discusses a set of facts in Luganda and Haya, two Bantu languages, which pose a problem for this latter sort of account. These languages display patterns of 'non-iterable' symmetry in passives, as described in the latter part of $\S 2$. Essentially, what we see is that an argument is able to move across no more than one other in the passive of these languages,

*Thanks to Michael Yoshitaka Erlewine, Colin Davis, David Pesetsky, and a helpful audience at Meiji Gakuin University for discussion of this project. Author: Kenyon Branan, National University of Singapore (kgbranan@nus.edu.sg) 
which I show to be unexpected for theories like those developed in McGinnis (1998); Doggett (2004). I suggest that this is reason to reject this sort of analysis. I then propose that these patterns are better accounted for under a theory where elements that-for independent reasonscannot undergo movement to a particular position are irrelevant for the evaluation of Shortest, which I spell out in more detail in $\S 3$. In $\S 4$ I discuss some patterns in English which are analogous to those discussed in Luganda and Haya, and sketch an extension of the account. $\S 5$ recaps and concludes.

\section{The background, the facts $\&$ the problem.}

2.1 THE BACKGROUND. In many languages, when there are two elements which could potentially move to a position in the clause, only one actually can. The English example in (1), involving a passive of a ditransitive, illustrates this. Here there are two elements which meet many of the conditions for being promoted to subject position: both are nominals, and neither is inherently licensed by an overt element like a preposition that might disqualify it for promotion. Nonetheless, the first object may be promoted, (1a), but the second may not, (1b).

(1) a. John was shown a picture.

b. * A picture was shown John

A common approach to patterns like (1) is to propose a principle like (2), which I will state quite informally here.

\section{(2) Shortest $^{1}$}

Given two potential movement operations, prefer the 'shortest' one.

On this view, (1) is explained by (2). Movement of the first object to subject position is in some sense shorter than movement of the second object, and, given Shortest, this will preclude movement of the second object to the subject position across the first.

Not all languages behave like English. Some, like Luganda, allow either internal arguments to be promoted to the canonical subject position in a passive, as we see in (3). Languages like Luganda are often described as having 'symmetric' passives, as opposed to the English passive, which is said to be 'asymmetric'.

\section{(3) An apparent violation of Shortest}

a. abaana ba-a-w-ebw' ekitabo

2.child 2-PST-give-PASS 7.book

'The children were given the book.'

b. ekitabo ky-a-w-ebw' abaana

7.book 7-PST-give-PASS 2.child

'The book was given to the children.'

The facts in (3) pose a challenge for theories of asymmetric passives that make use of Shortest. If the Luganda passive is like the English passive, then we should expect promotion of the second object to be blocked, since promotion of the first object is possible.

\footnotetext{
${ }^{1}$ For an exploration of different definitions of Shortest, see Fitzpatrick (2002).
} 
The leading proposal to capture the difference between English and Luganda while maintaining Shortest: a sequence of derivational steps alternatively called 'leapfrogging' [McGinnis (1998)] or 'hurdling' [Doggett (2004)] is responsible for the derivation of (3b). The difference between Luganda on the one hand and English on the other is that Luganda would allow the derivational sequence in (4), whereas English would not. The basic idea behind these proposals is that two instances of Shortest-respecting movement can give the appearance of a surface violation of Shortest. Movement of the lower object across the higher is contingent on movement of the lower object to the outer specifier of the phrase of which the higher object is also a specifier, as in (3a). This respects (a particular definition of) Shortest, since the lower object is the only element that could potentially move to the relevant position-for McGinnis (1998) a specifier cannot move to (another) specifier position of the phrase it is a specifier of; for Doggett (2004) a specifier is not in the search space of the head therefore is not a possible target for movement. Movement of the lower object to this position feeds movement to the actual landing site. Given (a particular definition of) Shortest, outer specifiers are closer to higher positions than inner specifiers; movement of the lower object to the terminal landing site from this intermediate position therefore respects Shortest, as in (3b).

\section{(4) Leapfrogging/hurdling}

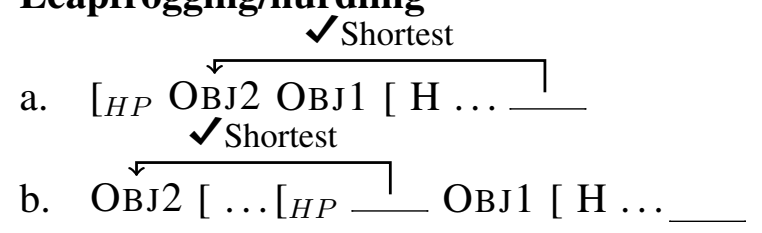

The theory here is analogous to theories of long-distance $\overline{\mathrm{A}}$-movement in a system where $\overline{\mathrm{A}}$-movement is required not to be too long. An important part of this sort of theory is that we expect this sort of movement to be unbounded. In much the same way that one intermediate step of movement may feed another in the case of long-distance $\bar{A}$-movement-necessary to capture the fact that this sort of movement may cross an arbitrary number of clause boundarieswe should expect one instance of leapfrogging to feed another. Successive cyclic movement of a lower argument to the specifier of a phrase hosting successively higher arguments should allow unbounded leapfrogging.

This is schematized abstractly in (5). Suppose a language has three elements, $\alpha, \beta$, and $\gamma$, all of which can potentially move to some position. If $\beta$ is structurally lower than $\alpha$, but is able to move across it, as in (5a), this indicates on the view we are considering that the leapfrogging derivation is available here. Similarly, (5b), if $\gamma$ is structurally lower than $\beta$, and is able to move across it, this indicates that the leapfrogging derivation is available here as well. When $\alpha, \beta$ and $\gamma$ are all in the same structure and compete for movement to the same position as in (5a-b), we expect two patterns of interest. Given (5a), we expect movement of $\beta$ to be able to cross $\alpha$ in this context, (5c). Likewise, given (5a-b), we expect movement of $\gamma$ to be able to cross both $\beta$ and $\gamma$ : the leapfrogging step in $(5 \mathrm{~b})$ should feed another step of leapfrogging analogous to (5a), allowing $\gamma$ to skip two elements. 


\section{(5) An expected pattern}

Given: $\sqrt{ }$ Shortest

a. $\quad \beta[\ldots \alpha \ldots$

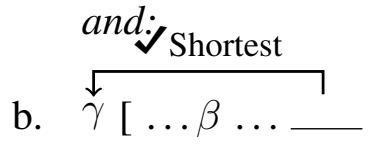

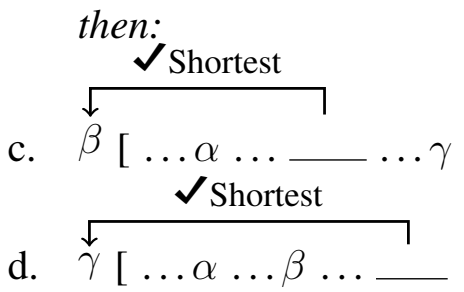

In other words: we expect this sort of symmetry to be iterable. If two elements behave symmetrically in pairs, then when these pairs are considered in tandem, all elements involve should behave symmetrically with respect to each other. Since this sort of theory does not impose any restrictions on the number of intermediate movement steps involved in the case of leapfrogging, we should in fact be surprised to find asymmetry arising in cases such as these.

2.2 THE FACTS. This is not the case-some languages, such as Luganda and Haya-appear to not allow the sort of passivization in $(5 \mathrm{~d})$, even though they have structures like (5a-c). To begin, consider (6-7). Here we see that Luganda and Haya have symmetric passives.

(6) Luganda and Haya have symmetric passives

a. abaana ba-a-w-ebw' ekitabo

2.child 2-PST-give-PASS 7.book

'The children were given the book.'

b. ekitabo ky-a-w-ebw' abaana

7.book 7-PST-give-PASS 2.child

'The book was given to the children.'

Luganda, Pak (2008), ex. 26a-b
a. ómawáana a-ha-háá-bwa
child
he-PST-give-PASS
b. ekitabo kí-ha-háá-bwa ómawáana
book it-PST-give-PASS child

ekitabo

'The book was given to the child.' Haya, Duranti \& Byarushengo (1977), ex. 83 [adapted]

'The book was given to the child.'

Haya, Duranti \& Byarushengo (1977), ex. 90

As we see in (8), this is true also of derived ditransitives-here, benefactive applicatives of underlyingly transitive verbs.

\section{(8) These passives are generally symmetric}

a. omusawo y-a-kwat-ir-w-a

1.doctor 1-PST-hold-APPL-PASS-fv 5.medicine

'The doctor had the medicine held for him.'

b. eddagala ly-a-kwat-ir-w-a omusawo

5.medicine 5-PST-hold-APPL-PASS-fv 1.doctor

'The medicine was held for the doctor.'

Luganda, Pak (2008), ex. 6a-b 
(9)
a. abáana bá-ka-cumb-il-w' ébitook
child they-PST-cook-APPL-PASS banana
'The children were cooked bananas.'

b. ebitook ki-ka-cumb-il-w

Haya, Duranti \& Byarushengo (1977), ex. 67 [adapted]

banana they-PST-cook-APPL-PASS child

'Bananas were cooked for the children.'

Haya, Duranti \& Byarushengo (1977), ex. 103 [adapted]

Postverbal agents in both Luganda and Haya may appear without a prepositional licensor, as we see in (10). The direct object in these cases may cross the postverbal agent.

\section{(10) Passive subjects may cross a postverbal agent}

a. ekinnyanja ky-a-fuumb-ibwa Nakato

7.fish 7-PST-cook-PASS N.

'The fish was cooked by Nakato' Luganda, Pak (2008), ex. 19

b. ebitooke bi-ka-cumb-w' ómukâzi

bananas they-PAST-cook-PASS woman

'The bananas were cooked by the woman.'

Haya, Duranti \& Byarushengo (1977), ex. 6

Postverbal agents may also be crossed by applicative arguments.
Passive subjects that were underlyingly applicative may cross a postverbal agent
a. ? omusawo y-a-wandiik-ir-wa
Mukasa ebbaluwa
1.doctor 1-PAST-write-APPL-PASS 1.Mukasa
9.letter
'The doctor was written a letter by Mukasa'
b. abáana bá-ka-cumb-il-w' ómukázy _ébitook
child they-PST-cook-APPL-PASS woman banana
'The children were cooked bananas by the woman.'

Haya, Duranti \& Byarushengo (1977), ex. 67

In other words, we see that there are a number of cases in Luganda and Haya where one nominal may cross another in the passive. Under an approach like that schematized in $\S 2$, this should indicate that the leapfrogging derivation is available for a variety of configurations of postverbal arguments.

Combining these symmetric configurations, however, causes asymmetry to arise, as we see below. (12a-b) show us that—as expected—an applicative can be passivized, and that the underlying indirect object may cross the instrumental applicative in these derived tetravalent configurations. We have seen before that direct objects may generally cross indirect objects in passive constructions in Luganda, as in (6-7). Likewise, we know that direct objects may cross applicatives, as shown in (8-9). But—unexpectedly-the direct object cannot cross both an indirect object and an applicative, as we see in (12c). 
(12) Stacking configurations causes asymmetry to arise
a. omuggo gw-a-lag-is-ibw-a omusomesa abaana
3.stick 3-PST-show-APPL-PASS-FV
1.teacher
2.child

"A stick was used to show the children the teacher."

b. omusomesa y-a-lag-is-ibw-a omuggo

Luganda, Pak (2008), ex. 9c

1.teacher 1-PST-show-APPL-PASS-FV 3.stick abaana

"The teacher was shown the children using a stick." 2.child
c. * abaana ba-a-lag-is-ibw-a omuggo omusomesa
2.child 2-PST-show-APPL-PASS-FV 3.stick 1.teacher

Luganda, Pak (2008), ex. $9 b$

"The children were shown to the teacher using a stick."

Luganda, Pak (2008), ex. 9d

This is unexpected on a view where symmetric passives are attributed to the hurdling/leapfrogging sketched in the previous section. For this approach, there's no way to rule out multiple instances of hurdling. ${ }^{2}$ We expect the lowest argument to be able to hurdle first over the medial argument followed by hurdling over the leftmost/highest argument, giving us the [unattested] (12c).

Note that this property of passives is not specific to underlying ditransitives, but seems to be a general property of passives in which three nominals underlyingly appear post-verbally. This is shown below with passives of non-derived ditransitives with an overt postverbal agent.

(13) The stacking effect holds more generally

a. abaana ba-a-w-ebw' omusajja ekitabo 2.child 2-PST-give-PASS 1.man 7.book

'The children were given the book by the man.'

b. * ekitabo ky-a-w-ebw' omusajja abaana 7.book 7-PST-give-PASS 1.man 2.child

'The book was given to the children by the man.' Luganda, Pak (2008), ex. 26a-b
a. omwáán' a-ka-háá-bw-a kat' ekitabo
child he-PST-give-PASS-FV K. book
'The child was given a book by Kato'
b. * ekitabo kí-ka-háá-bw-a kat'omwáán' book he-PST-give-PASS-FV K. child 'The book was given to the child by Kato.'

Haya, Duranti \& Byarushengo (1977), ex. 83, 86

The problem here is comparable to that which we saw before. We know from earlier that in Luganda and Haya, direct objects are able to 'skip' indirect objects and overt agents, considered in isolation. Likewise, (13a) and (14a) show us that indirect objects are able to skip overt agent We should therefore expect direct objects to be able to skip both an indirect object and

\footnotetext{
${ }^{2}$ See Pak (2008) for a discussion of some possibilities [and the difficulties they face].
} 
overt agent, through sequential hurdling operations.

2.3 THE PROBLEM. The theory of symmetry in A-movement discussed in $\S 2$ makes use of a mechanism analogous to intermediate movement in cases of long-distance $\bar{A}$-movement. In the cases we have just seen, this analogy breaks down, as schematized below. In cases of longdistance movement, (15a) there are an unbounded number of intermediate movement stepsminimally at the edge of every clause that the wh-phrase passes through, and perhaps more if $\mathrm{vP}$ is a phase. On the other hand, in the putative hurdling cases, (15), we see that there seem to be a bounded number of intermediate movement steps.

\section{(15) The analogy breaks down}

a. Successive cyclic movement:

$\checkmark$ Who [ did Alice say [whe that Beth thinks [who that Charles met who

b. Hurdling:

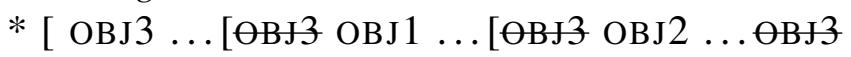

These are patterns of non-iterable symmetry, which hurdling leads us not to expect. To account for them we will need a mechanism to allow one nominal to skip another, but it will be need to be restricted in a way that hurdling is not: it cannot be 'used' more than once in a given derivation.

3. An analysis. Having seen in the previous section that theories of symmetry that make use of intermediate movement steps make undesirable predictions, I will in this section develop an alternative. This theory will make use of a notion of 'conflict resolution', in which locality restrictions may be ignored if satisfying them would result in the violation of some other locality requirement. Crucially, as we will see, this theory naturally leads us to expect the patterns of non-iterable symmetry in Luganda and Haya.

3.1 THE BASIC IDEA. This theory will maintain Shortest, repeated in (16). To maintain Shortest while explaining the basic Luganda and Haya facts, we will need some method of allowing one argument to cross another.

\section{Shortest}

Given two potential movement operations, prefer the shortest one.

The novel proposal consists of a principle of conflict resolution, which will allow (16) to be violated in certain circumstances. Rather than posit steps of intermediate movement which give rise to apparent violations of Shortest, I suggest that elements may be ignored for the evaluation of Shortest.

Principle of Conflicting Requirements

Elements don't count for Shortest if their movement would violate (17).

(18) is a ban on movement which is in some sense 'too short'. See Bošković (1997, 2016), Erlewine (2016), and Deal (to appear), among others, for proposals of a similar nature. ${ }^{3}$ It rules

\footnotetext{
${ }^{3}$ This is not an exhaustive list of theories of anti-locality. A number of spiritually similar proposals have been made in Murasugi \& Saito (1994); Ishii (1997); Bošković (1997); Saito \& Murasugi (1999); Abels (2003); Grohmann (2003)
} 
out movement from one specifier to the 'next specifier up'.

\section{(18) Generalized spec-to-spec anti-locality}

a. Movement from XP must cross a maximal projection other than XP.

b. Movement from $\mathrm{A}$ to $\mathrm{B}$ crosses $\mathrm{C}$ iff $\mathrm{C}$ dominates $\mathrm{A}$ but not $\mathrm{B}$.

Putting these proposals together allows us to understand why one element may cross another in languages like Luganda and Haya. On this view, apparent violations of Shortest do not (necessarily) reflect an intermediate step of movement that brings one nominal closer to the final landing site than another. When two arguments could potentially move to a target poistion, the lower is preferred over the higher just in cases where the landing site is the 'next specifier up' for the higher element. Otherwise the closest argument wins. A crucial feature of this theory is that, for a given position, there can be one and only one element which is 'anti-local' to it. This leads us to expect the patterns of non-interative symmetry demonstrated in Luganda and Haya. The following subsection spells out an account in more detail.

3.2 How IT WORKS. Assume a structure for the passive clause in Luganda and Haya like that in (19). Assume also that the head $\mathrm{v}_{\text {pass }}$ bears an EPP feature that might be satisfied in two ways: either through external merge of an agent, or by movement of an internal argument to its specifier. Note, crucially, that movement from spec-vP to spec-TP here will violate antilocality. ${ }^{4}$

\section{Luganda/Haya (passive) clause structure

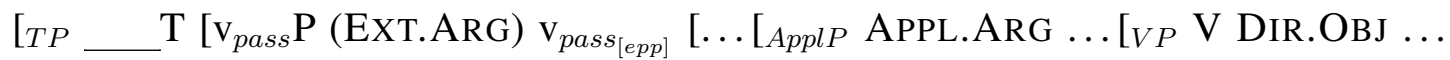

We will begin with a simple case, which illustrates how one argument may be skipped by another. Recall that post-verbal agents may generally be skipped by the highest internal argument, shown once more in (20-21).

\section{(20) Conflicting requirements approach predicts symmetry between two highest argu-} ments
a. ? omusawo y-a-wandiik-ir-wa
Mukasa
1.doctor 1-PAST-write-APPL-PASS M. ebbaluwa
'The doctor was written a letter by Mukasa'
b. abáana bá-ka-cumb-il-w’ ómukázy ébitook
child they-PST-cook-APPL-PASS woman banana
'The children were cooked bananas by the woman.'

Haya, Duranti \& Byarushengo (1977), ex. 67

\footnotetext{
${ }^{4}$ This would suggest that the structure of non-passive clauses is different in some way than that of passives clauses, so as to allow the external argument to move to the canonical subject position. One possibility is that there is an additional functional projection between $\mathrm{T}$ and $\mathrm{v}$ in active clauses; the presence of this projection will allow movement from spec-vP to spec-TP to respect anti-locality. Another possibility is that the subject in active clauses in Luganda and Haya occupies a higher position than the subject in passive clauses—say, spec-CP, as opposed to spec-TP.
} 

a. ekinnyanja ky-a-fuumb-ibwa Nakato
7.fish 7-PST-cook-PST N.
'The fish was cooked by Nakato'
Luganda, Pak (2008), ex. 19
b. ebitooke bi-ka-cumb-w' ómukâzi
bananas they-PAST-cook-PASS woman
'The bananas were cooked by the woman.'

Haya, Duranti \& Byarushengo (1977), ex. 6

(20-21) will have structures like (22). The external argument occupies spec-vP. It therefore cannot move to spec-TP, since doing so would violate anti-locality. Movement of the highest internal argument thus respects Shortest—it is the shortest move that can possibly be made.

\section{(22) Skipping an external argument}

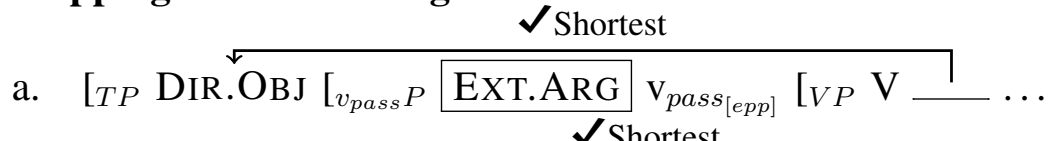

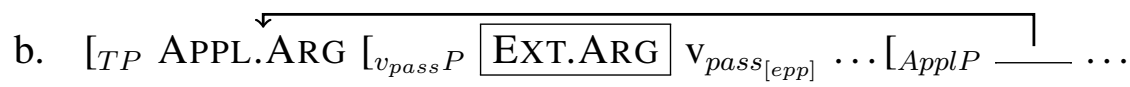

Likewise, this allows us to understand why symmetry arises when the agent is not overt. If the indirect object moves to Spec- $\mathrm{v}_{\text {pass }} \mathrm{P}$, as schematized in (23b), it will not be considered for the evaluation of Shortest. When this position is not filled by an external argument-either overt or phonologically null-it must be filled by an internal argument. In cases where there are two (or more) internal arguments, it must be the closest one that moves to this position. Movement of the higher object to this position means that it cannot undergo further movement to the canonical subject position, since this would result in a violation of anti-locality. This allows the lower object to move across it without violating Shortest.

\section{(23) Accounting for symmetry in agentless passives}

a. ekitabo ky-a-w-ebw' abaana

7.book 7-PST-give-PASS 2.child

'The book was given to the children.'

Luganda, Pak (2008), ex. $26 b$

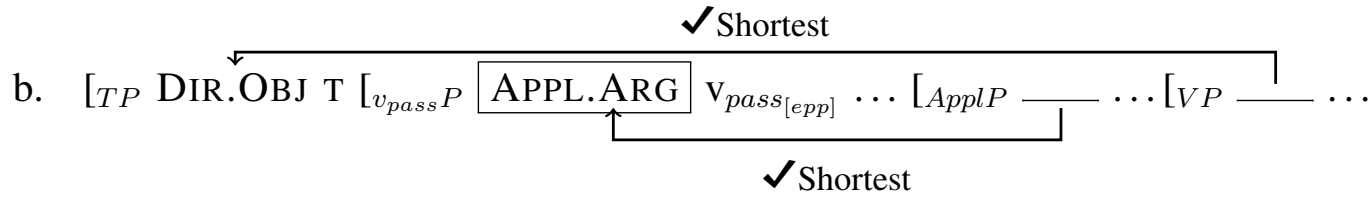

Finally, this system allows us to understand why one-and only one-nominal may be skipped, shown once more below.

\section{The problematic pattern of non-iterative symmetry}

*ekitabo ky-a-w-ebw' omusajja abaana

7.book 7-PST-give-PASS 1.man 2.child

'The book was given to the children by the man.'

Luganda, Pak (2008) ex. $26 b$

This is simply a violation of Shortest, as sketched below. Spec,vP is filled by the external ar- 
gument. This precludes movement of the external argument to the subject position, since doing so would violate anti-locality, thereby allowing the higher internal argument to move across the external argument. It also has the effect of trapping the lower internal argument in-situ. The medial argument cannot move to the singular position that would allow it to be bypassed, since that position is already occupied. Nothing precludes movement of the medial argument to subject position, so Shortest blocks movement of the lower argument in this case. Since there is one [and only one] position in the clause which is anti-local to the canonical subject position, one and only one nominal may be 'skipped' in the passive.

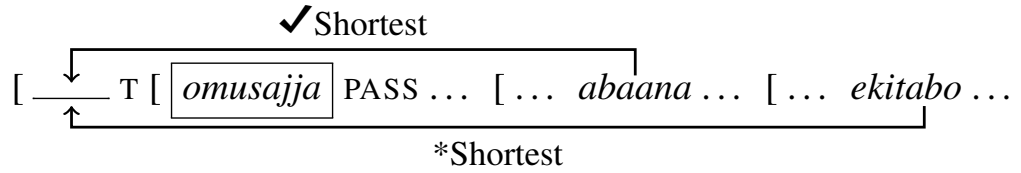

Derivations of the sort in (25) also allow us to understand why movement of the lower argument across the higher is not obligatory when the agent is not overtly expressed. Recall here that I suggested that a phonologically null agent might satisfy the EPP feature of $\mathrm{v}_{\text {pass }}$. In such cases, the highest internal argument will move to the canonical subject position, as a result of Shortest.

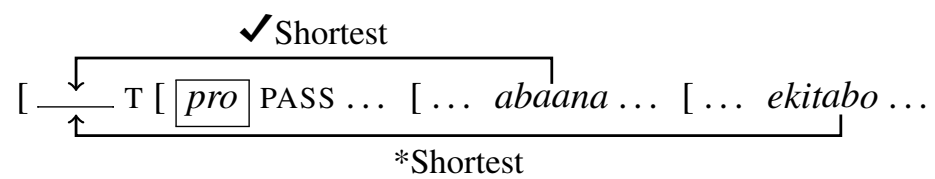

If this is on the right track, then there's a structural difference between (27a-b) under this analysis. (27a) has an phonologically null agent in spec,vP. (27b) lacks an agent altogether.

\section{Luganda and Haya have symmetric passives}

a. abaana ba-a-w-ebw' [vP pro ekitabo

2.child 2-PST-give-PASS 7.book

'The children were given the book.'

b. ekitabo ky-a-w-ebw' [vP abaana

7.book 7-PST-give-PASS 2.child

'The book was given to the children.' Luganda, Pak (2008), ex. 26a-b

Evidence for this difference - at least in Luganda - comes from an interpretive asymmetry involving agent-oriented adverbs in these sorts of passives. (28a) has a phonologically null agent in spec,vP, and thus allows an agent oriented adverb. (27b) lacks an agent altogether, with the higher argument occupying spec,vP and doesn't allow an agent oriented adverb.

\section{(28) Theme passives cannot be agentive}

a. akamyu ka-a-lis-ibw-a [ kasooli n'obwegendereza

12.rabbit 12-PST-feed-PASS-FV 1.corn with.care

'The rabbit was fed corn with care.'

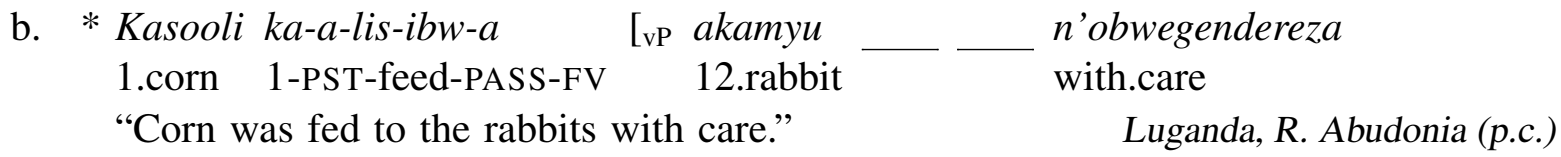


Patterns of non-iterable symmetry pose a problem for leapfrogging/hurdling approaches to symmetric passives. I proposed a different sort of solution: elements that can't move because of anti-locality don't count for the evaluation of Shortest. This has a desirable result: one and only one nominal may be skipped by another, since there's only one position in the clause that's anti-local to another.

4. English extensions. In this section I will attempt to extend the theory developed in the previous sections to some English patterns. To begin we will consider the choice of fronted PP in cases of locative inversion in which multiple locatives appear postverbally. Consider first the sentences in (29), illustrating that two different sorts of PP-those designating a 'path' of movement, and those designating the 'endpoint' of movement—may be fronted in the locative inversion construction.

(29) Locative inversion with paths and endpoints

a. Down the hill rolled the baby carriage.

b. Into the lake rolled the baby carriage.

(30) shows us that these PPs have a fixed order when they appear in the same clause. The path must precede the endpoint.

(30) No inversion of paths and endpoints

a. The baby carriage rolled [down the hill] [into the lake].

b. * The baby carriage rolled [into the lake] [down the hill].

Variable binding suggests that the path c-commands the endpoint, but not vice-versa.

\section{(31) Asymmetric command relationship between path and endpoint}

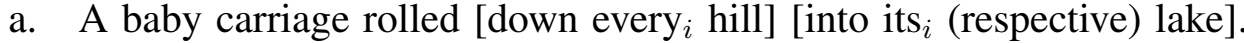

b. * A baby carriage rolled [down its $_{i}$ (respective) hill] [into every ${ }_{i}$ lake].

The configuration here is reminiscent of the patterns in Luganda and Haya examined earlier in this paper. Here we see a similar pattern. In (30) we saw that paths and endpoints are potential targets for locative inversion. Interestingly, as we see in (32b), endpoints cannot invert across both a path and the nominal argument.

\section{(32) Endpoints may not invert in the presence of a path}

b. * Into the lake rolled the baby carriage down the hill

a. Down the hill rolled the baby carriage into the lake.

The theory sketched in $\S 3.2$ could be carried forward to these patterns. On this view, locative inversion would involve movement of the internal argument, baby carriage, to a position antilocal to spec,TP; movement to this position allows the PP to bypass the argument and move to spec,TP. When both the path and endpoint are present in the structure, movement of the former blocks movement of the latter.

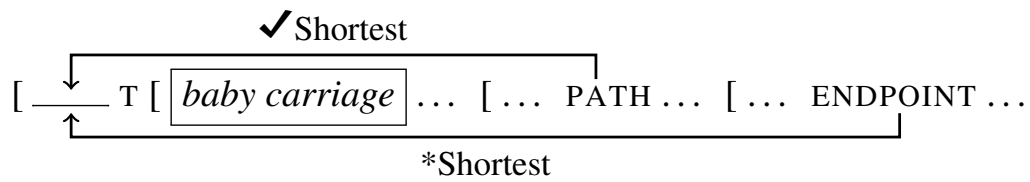


Another potential application of this theory is to the pseudopassive. In English, prepositional arguments of certain verbs may strand the preposition in the passive, (34b) but the PP as a whole may not be promoted to subject position. Note that this is somewhat mysterious given our discussion of locative inversion just above - there we saw that there is nothing in particular in English which precludes movement of a PP to the canonical subject position.

\section{(34) Pseudopassives}

a. Morpho spoke to Hoppy.

b. Hoppy was spoken to

c. * To Hoppy was spoken

I would like to suggest that we can see this in a similar sense to the cases of theme passives in Luganda and Haya. There we saw that movement of an argument to a particular position may allow it to be ignored for the evaluation of Shortest to a higher position. What I would like to suggest is that the same thing happens in the case of the pseudopassive: movement of the PP to a particular position feeds subextraction of the DP within it.

Consider first the fact that these arguments display variable order with other elements in VP, like manner adverbs, (35). One interpretation of these facts is that there are at least two positions in VP which these arguments can occupy: one above the adverb, and one below.

\section{(35) PPs display variable order}

a. Morpho spoke loudly to Hoppy.

b. Morpho spoke to Hoppy loudly.

Note now that in the pseudopassive, the stranded preposition must occupy the leftmost or higher position, (36). (37) simply shows us that it is in principle possible to strand the preposition after the adverb. This suggests that the unavailability of stranding in (36b) is not a more general fact about the stranding of prepositions more generally.

\section{Stranded $P$ must be verb adjacent}

a. Hoppy was spoken to loudly (by Morpho)

b. * Hoppy was spoken loudly to _ (by Morpho)

a. Who did Morpho speak to loudly?

b. Who did Morpho speak loudly to

We can think of this as another case of movement of one element to an anti-local position feeding movement of another element, analogous to the analysis of theme passives in $\S 4$. We have seen that the PPs in question display a relatively free distribution in the post-verbal field. I would like to suggest that this is not the case in passives: these PPs are forced to move to a position high in the post-verbal field, schematized below. This movement precludes further movement of the PP to spec,TP. Movement of the DP inside the PP, however, is allowed as a result: the PP is ignored for the evaluation of Shortest for movement to spec,TP as a result of anti-locality, so movement of the DP dominated by PP to spec,TP is allowed.

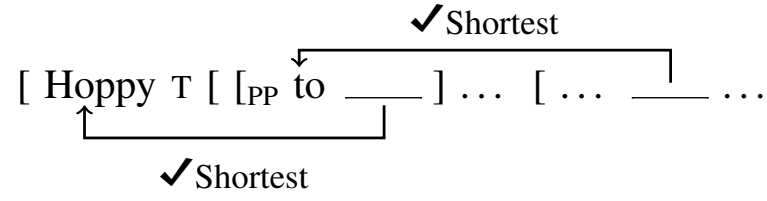


What we see here, more broadly, is that the theory is not too finely tuned for the cases we investigated. A more familiar language, English, displays patterns that we expect given the theory we have developed. Here we have seen that locative inversion in English is subject to an identical restriction as the passive in Luganda and Haya: a postverbal argument may pass no more than one other argument on its way to the canonical subject position. We have seen also that the pseudopassive is amenable to analysis as a case of movement to an anti-local position as a mechanism by which an argument might be bypassed.

5. Recap and conclusion. In this paper I noted a pattern of asymmetry in passives in Luganda and Haya that pose a problem for leading theories of symmetry in passives. We saw that symmetry is non-iterative in these languages. In other words, the mechanism that allows one nominal to be promoted 'across' another is restricted, in that it can apply no more than once per clause. I suggested that this was strong enough to reject such theories, and proposed a different theory of symmetry in passives. This theory makes use of the notion of conflict resolution; on this view a lower nominal may move to a particular landings 'across' a higher nominal in cases where the higher nominal is too close to the landing site to itself undergo movement. I showed that this correctly derives the Luganda and Haya patterns, and then noted some possible extensions of the account to locative inversion and the pseudopassive in English.

\section{References}

Abels, Klaus. 2003. Successive cyclicity, anti-locality, and adposition stranding: University of Connecticut dissertation.

Bošković, Željko. 1997. The syntax of nonfinite complementation: An economy approach 32. MIT Press.

Bošković, Željko. 2016. Getting really edgy: On the edge of the edge. Linguistic Inquiry 47(1). 1-33. https://doi.org/10.1162/LING a_00203.

Chomsky, Noam. 1973. Conditions on transformations. In Steven Anderson \& Paul Kiparsky (eds.), A festschrift for Morris Halle. 232-286. Holt, Rinehart and Winston.

Deal, Amy Rose. to appear. Raising to ergative: remarks on applicatives of unaccusatives. Linguistic Inquiry 50:2. https://doi.org/10.1162/ling a_00310.

Doggett, Teal Bissell. 2004. All things being unequal: Locality in movement: Massachusetts Institute of Technology dissertation.

Duranti, Alessandro \& Ernest Byarushengo. 1977. On the notion of 'direct object'. Haya Grammatical Structures, Southern California Occasional Papers in Linguistics 6. 45-71.

Erlewine, Michael Yoshitaka. 2016. Anti-locality and optimality in Kaqchikel Agent Focus. Natural Language \& Linguistic Theory 34(2). 429-479.

Fitzpatrick, Justin M. 2002. On minimalist approaches to the locality of movement. Linguistic Inquiry 33(3). 443-463. https://doi.org/10.1162/002438902760168563.

Grohmann, Kleanthes K. 2003. Prolific domains: On the anti-locality of movement dependencies, vol. 66 Linguistik Aktuell. Amsterdam: John Benjamins Publishing. https://doi.org/10.1075/la.66.

Ishii, Toru. 1997. An asymmetry in the composition of phrase structure and its consequences: University of California, Irvine dissertation.

McGinnis, Martha. 1998. Locality in A-movement: Massachusetts Institute of Technology dissertation.

Murasugi, Keiko \& Mamoru Saito. 1994. Adjunction and cyclicity. Proceedings of the West 
Coast Conference on Formal Linguistics (WCCFL) 13. 302-17.

Pak, Marjorie. 2007. A-movement: locality and intervention effects in Luganda. Handout, WCCFL talk.

Pak, Marjorie. 2008. A-movement and intervention effects in Luganda. Proceedings of the West Coast Conference on Formal Linguistics (WCCFL) 27. 361-369.

Saito, Mamoru \& Keiko Murasugi. 1999. Subject predication within IP and DP. In Kyle Johnson \& Ian Roberts (eds.), Beyond principles and parameters. 167-188. Berlin: Kluwer Academic Publishers. 